\title{
ORIGINAL ARTICLE DUOX1 silencing in lung cancer promotes EMT, cancer stem cell characteristics and invasive properties
}

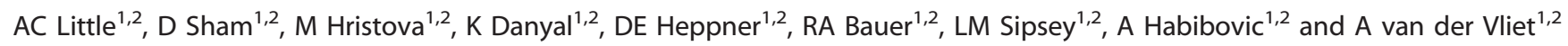

Dual oxidase 1 (DUOX1) is an oxidant-generating enzyme within the airway epithelium that participates in innate airway host defense and epithelial homeostasis. Recent studies indicate that DUOX1 is suppressed in lung cancers by epigenetic silencing, although the importance of DUOX1 silencing in lung cancer development or progression is unknown. Here we show that loss of DUOX1 expression in a panel of lung cancer cell lines is strongly associated with loss of the epithelial marker E-cadherin. Moreover, RNAi-mediated DUOX1 silencing in lung epithelial cells and the cancer cell line $\mathrm{NCl}-\mathrm{H} 292$ was found to result in loss of epithelial characteristics/molecular features (altered morphology, reduced barrier function and loss of E-cadherin) and increased mesenchymal features (increased migration, anchorage-independent growth and gain of vimentin/collagen), suggesting a direct contribution of DUOX1 silencing to epithelial-to-mesenchymal transition (EMT), an important feature of metastatic cancer. Conversely, overexpression of DUOX1 in A549 cells was capable of reversing EMT features. DUOX1 silencing in H292 cells also led to enhanced resistance to epidermal growth factor receptor tyrosine kinase inhibitors such as erlotinib, and enhanced levels of cancer stem cell (CSC) markers CD133 and ALDH1. Furthermore, acquired resistance of H292 cells to erlotinib resulted in enhanced EMT and CSC features, as well as loss of DUOX1. Finally, compared with control H292 cells, H292-shDUOX1 cells displayed enhanced invasive features in vitro and in vivo. Collectively, our findings indicate that DUOX1 silencing in lung epithelial cancer cells promotes features of EMT, and may be strongly associated with invasive and metastatic lung cancer.

Oncogenesis (2016) 5, e261; doi:10.1038/oncsis.2016.61; published online 3 October 2016

\section{INTRODUCTION}

The NADPH oxidase dual oxidase 1 (DUOX1) is highly expressed in differentiated airway and alveolar epithelia, and generates $\mathrm{H}_{2} \mathrm{O}_{2}$ in response to various environmental triggers as part of innate protective response against challenges to the airway. ${ }^{1-3}$ Activation of airway DUOX1 mediates epithelial production of inflammatory mediators and mucus proteins, and promotes cell migration, as critical events in mucosal host defense and maintenance of airway epithelial integrity, ${ }^{2,4,5}$ events that largely depend on redoxdependent activation of cell signaling mechanisms involving Src family tyrosine kinases ${ }^{6,7}$ and epidermal growth factor receptor (EGFR)-dependent pathways. ${ }^{4,7,8}$ As increased expression and/or activation of Src and EGFR are well-known features of lung cancer, ${ }^{9-11}$ it is plausible that lung cancer may also be associated with increased expression or activation of DUOX1. Indeed, production of reactive oxygen species (ROS) from NADPH oxidases (NOX), including DUOX1, has been reported to promote genomic instability and alter signaling pathways involved in carcinogenesis, ${ }^{12-14}$ and cancer cells commonly produce elevated levels of ROS as potential mediators of mitogenic signaling and metastasis. ${ }^{15-17}$ Furthermore, expression of certain NOX isoforms is often increased in various cancers, although the distribution and expression levels are highly diverse across cancers, and the biological roles of different NOX isoforms in cancer development and progression are still poorly understood. ${ }^{12,14}$

In apparent contrast with these concepts, several recent studies demonstrated that DUOX1, as well as its maturation factor, DUOXA1, is frequently silenced in various epithelial cancers, including lung cancer, due to DNA hypermethylation within their promoter regions. ${ }^{18-21}$ The significance of DUOX1/DUOXA1 silencing in cancer is unclear, and might suggest a role for DUOX1 and/or DUOXA1 as a potential tumor suppressor, potentially by mediating ROS-dependent inhibition of cytokinesis. ${ }^{22}$ DUOX1 suppression may also be relevant for maintenance of cancer stem cells (CSCs), which typically contain reduced ROS levels as an essential feature for maintaining quiescence and self-renewal. ${ }^{23,24}$ Furthermore, as DUOX1 activation participates in epithelial Src/EGFR-mediated signaling, ${ }^{6-8}$ DUOX1 silencing might result in altered regulation of these kinases, with potential consequences for development of resistance against tyrosine kinase inhibitors (TKIs). In this regard, responsiveness of non-small-cell lung cancers to EGFR TKI is strongly related to epithelial phenotype $e^{25-27}$ and acquired resistance to EGFR TKI has been associated with epithelial-tomesenchymal transition (EMT), a critical feature of invasive and metastatic cancers that is associated with poor prognosis in lung cancer. ${ }^{26,28-30}$

The present studies were conducted to address the potential association of DUOX1 silencing in lung cancer, with development of EMT and EGFR TKI resistance. Indeed, our results demonstrate that RNAi-mediated DUOX1 silencing in lung epithelial cells and the lung cancer cell line $\mathrm{H} 292$ induces loss of epithelial characteristics, increases features of EMT and promotes invasive properties. Conversely, DUOX1 overexpression in lung cancer cells was able to reverse EMT features and enhance epithelial characteristics. DUOX1 silencing was also found to promote EGFR

\footnotetext{
${ }^{1}$ Department of Pathology and Laboratory Medicine, University of Vermont, Burlington, VT, USA and ${ }^{2}$ Vermont Lung Center, College of Medicine, University of Vermont, Burlington, VT, USA. Correspondence: Dr A van der Vliet, Department of Pathology and Laboratory Medicine, University of Vermont, HSRF Building, Room 216, 149 Beaumont Avenue, Burlington, VT 05405, USA.

E-mail: albert.van-der-vliet@uvm.edu

Received 22 January 2016; revised 30 June 2016; accepted 1 August 2016
} 
TKI resistance and enhance features of CSCs, suggesting the significance of DUOX1 silencing in lung cancer as a possible indicator of lung cancers with poor prognosis or lack of responsiveness to common anticancer therapy.

\section{RESULTS}

DUOX1 silencing induces features of EMT

We evaluated a panel of non-cancer airway epithelial cells and various lung cancer cell lines for mRNA expression of DUOX1 and DUOX2, and observed a general loss of DUOX1 and DUOX2 in most lung cancer cell lines tested (Figure 1a; Supplementary
Figure $\mathrm{S} 1 \mathrm{~A}$ ), with the exception of the pulmonary mucoepidermoid cell line H292 and the lung adenocarcinoma cell line Calu-3, two cancer-derived cell lines that maintain many normal airway epithelial features ${ }^{8,31}$ and maintain DUOX1 expression. We therefore hypothesized that epithelial DUOX1 expression may associate with epithelial characteristics. Indeed, DUOX1 expression status in a panel of lung cancer cell lines strongly correlated with expression of the epithelial marker E-cadherin $(\mathrm{CDH} 1$, Figure 1a), suggesting that DUOX1 silencing in lung cancer is associated with loss of epithelial characteristics. To explore this further, we silenced DUOX1 in the DUOX1-expressing cell line H292, by stable transfection with DUOX1-targeted a

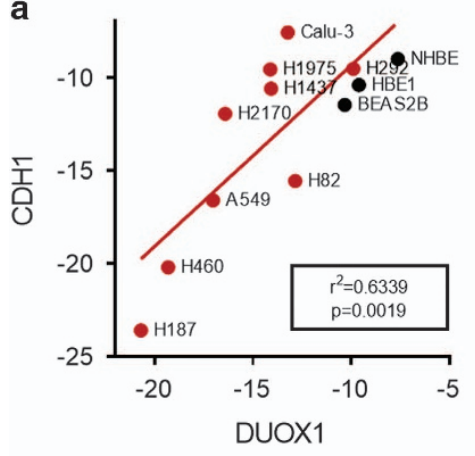

b

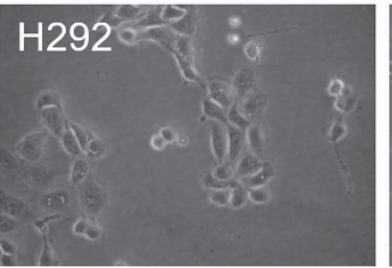

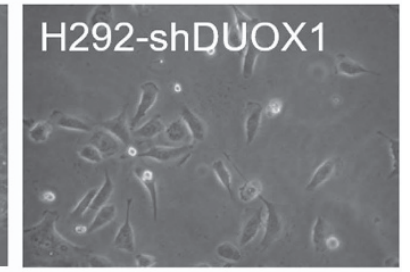

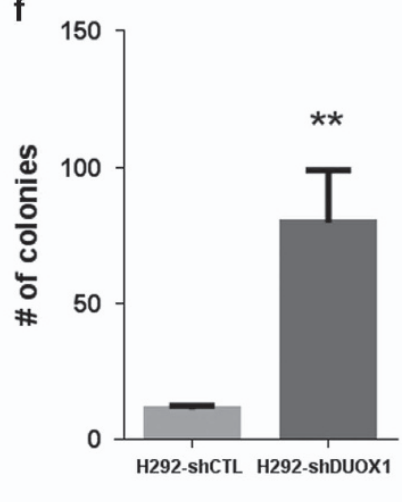

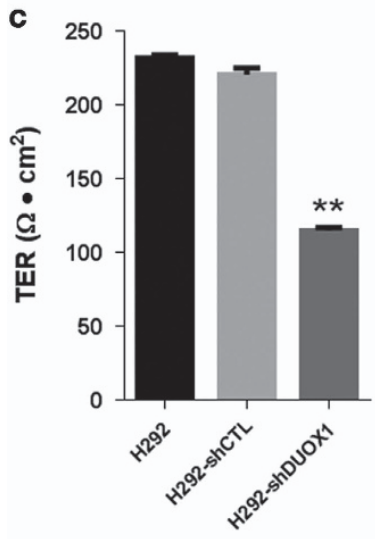

d
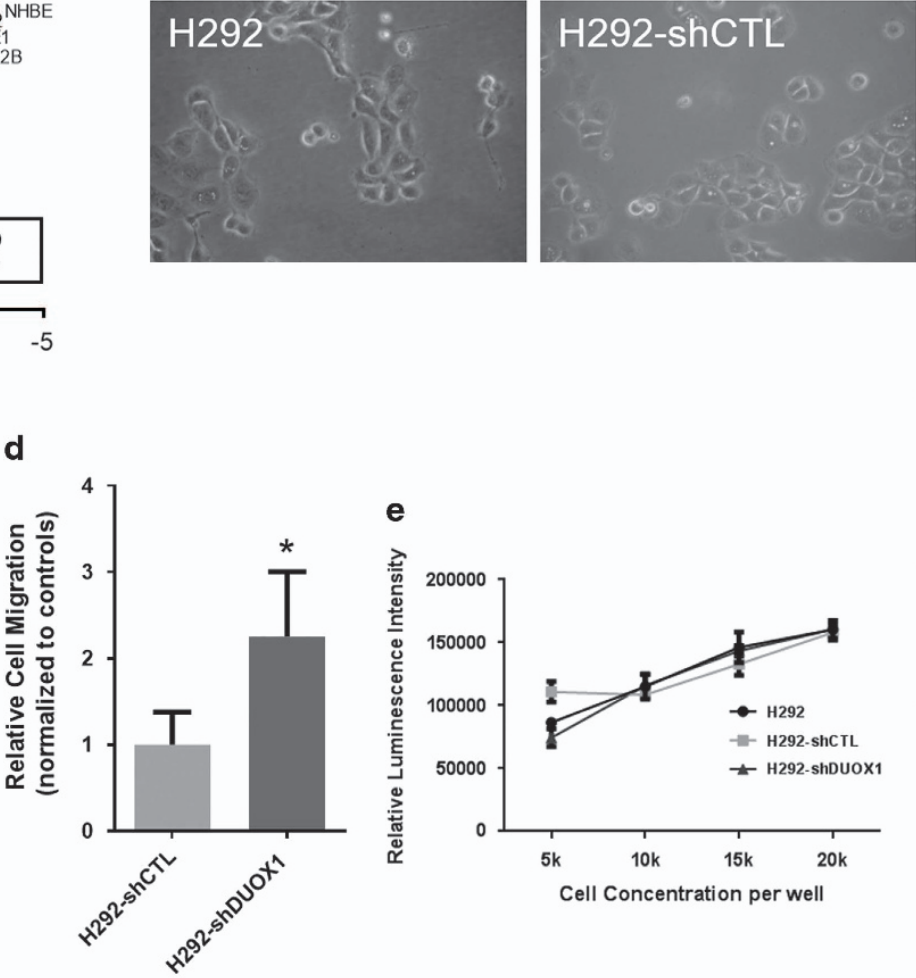

g
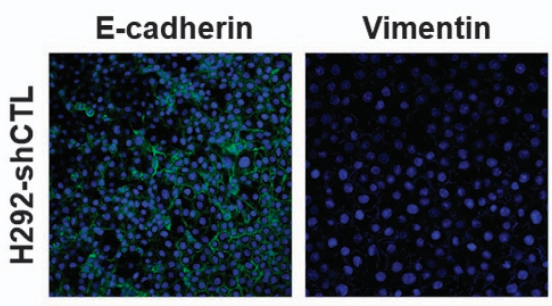

SMA
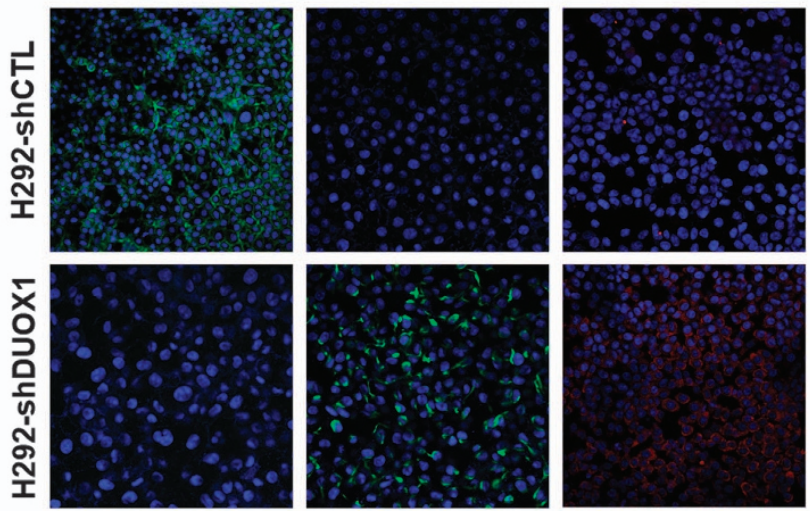

h

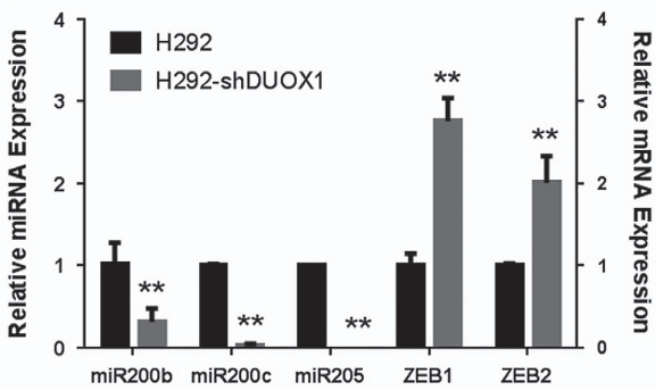

Figure 1. DUOX1 silencing displays functional mesenchymal features. (a) Evaluation of DUOX1 and E-cadherin (CDH1) mRNA expression in normal non-cancer airway epithelial cells (black dots) and a panel of lung cancer cell lines (red dots). (b) Phase contrast brightfield microscope images taken of host cell line H292, H292 cells transfected with an empty vector (H292-shCTL) and H292 cells transfected with short hairpin RNA targeted against DUOX1 (H292-shDUOX1). (c) Trans-epithelial resistance $(\Omega)$ characterization of each cell model. (d) Cell migration as measured by Donut cell migration assay. (e) Cells were seeded at various densities and allowed to grow in culture for $72 \mathrm{~h}$. Post $72 \mathrm{~h}$, cell viability via ATP production was determined in a luminescence assay. (f) Cells were grown on soft agar, and colonies counted to assess anchorage-independent growth. (g) Fluorescent images of EMT markers; SMA, smooth muscle actin. (h) Quantitative real-time PCR results of miR-200 family and transcriptional regulators of E-cadherin, ZEB1/2. ${ }^{*} P<0.05$ and ${ }^{* *} P<0.01$ were calculated by Student's $t$-test. 
a
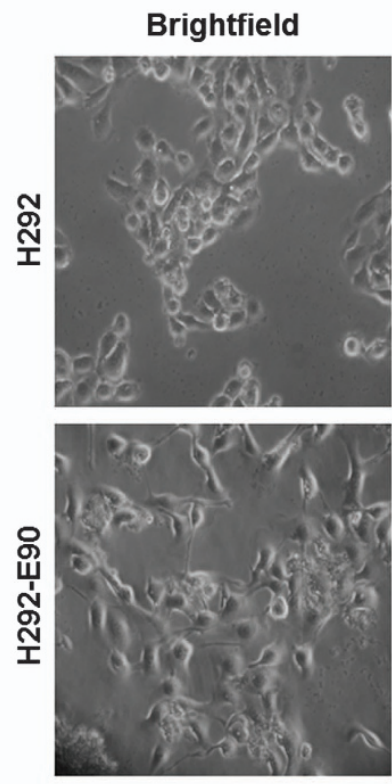

b

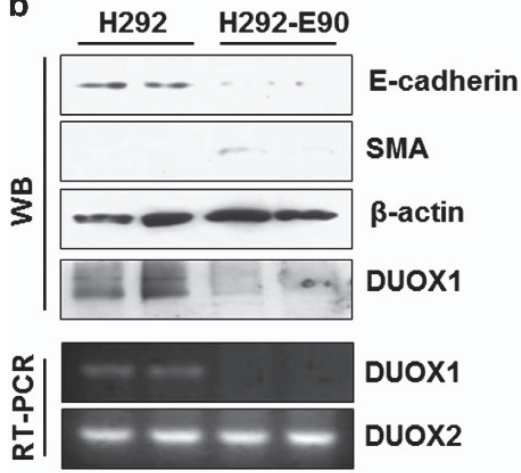

E-cadherin
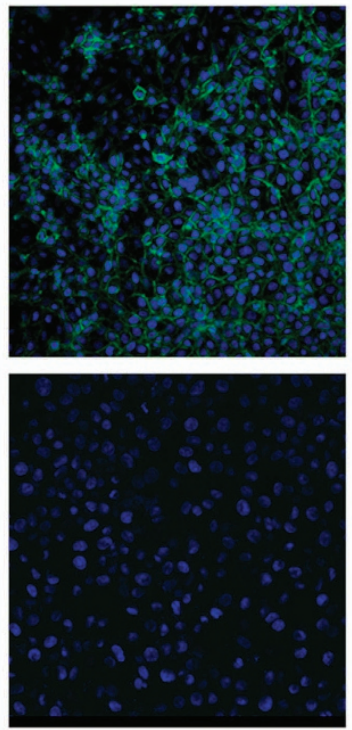
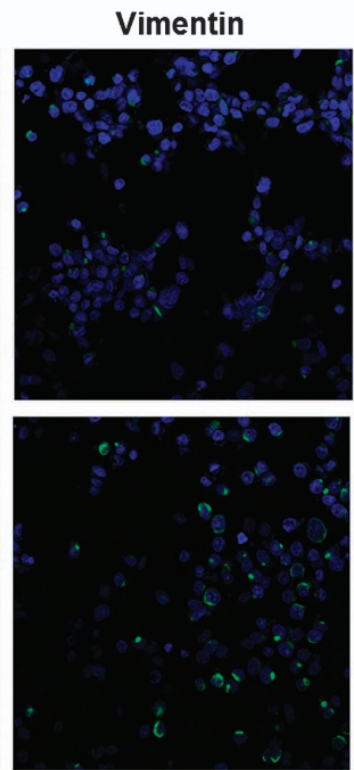

SMA
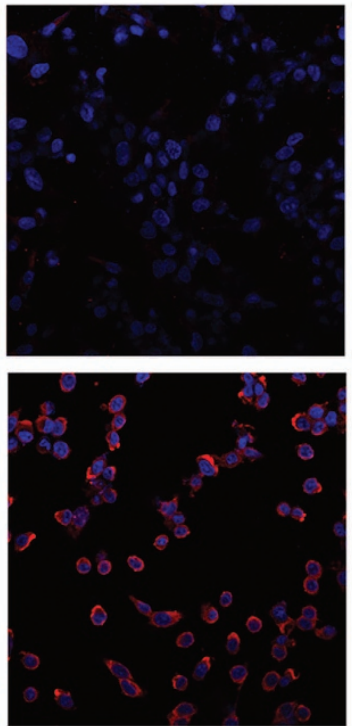

C

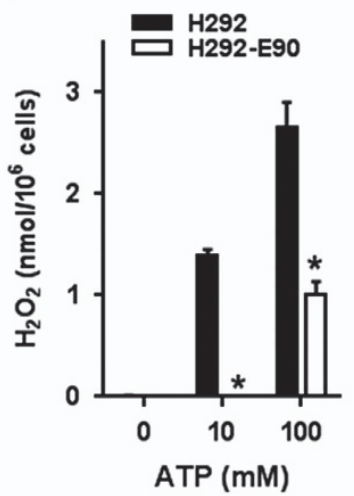

d

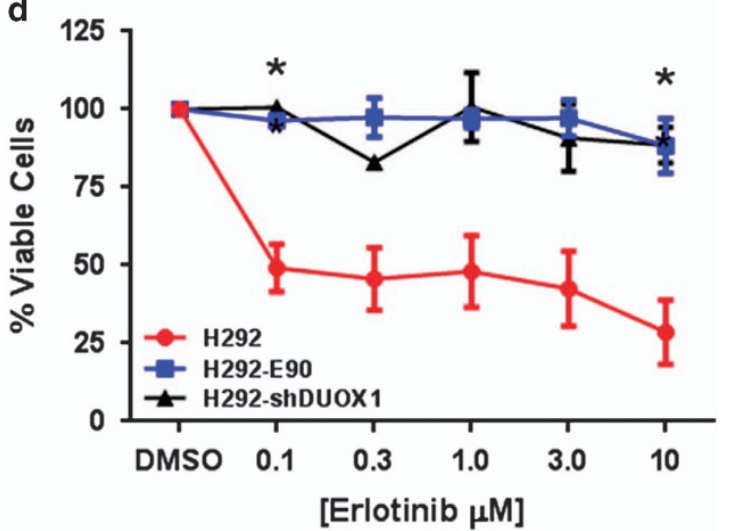

Figure 2. DUOX1 silencing is associated with acquired resistance to the EGFR TKI, erlotinib. (a) Brightfield and fluorescent confocal imaging of host cell line H292 and erlotinib-resistant H292 cell model (H292-E90) displaying features of EMT. (b) Protein and RNA analysis of EMT markers. (c) High-performance liquid chromatography $\mathrm{H}_{2} \mathrm{O}_{2}$ measurements of ATP-stimulated $\mathrm{H} 292$ and H292-E90 cell lines. (d) Percentage of viable cells post treatment of chemotherapeutic, erlotinib. ${ }^{*} P<0.05$ and ${ }^{* *} P<0.01$ were calculated by one-way analysis of variance or Student's $t$-test.

shRNA, ${ }^{7}$ and selected three individual clones for use in subsequent studies. We observed that H292-shDUOX1 cells display altered morphological features compared with normal or control transfected H292 cells (Figure 1b), with loss of cobblestone appearance and intercellular junctions, and a more fibroblast-like cell morphology. Functional analysis also indicated a loss of epithelial characteristics of H292-shDUOX1 cells, with markedly reduced transepithelial electrical resistance (Figure 1c), reflecting loss of epithelial barrier function due to loss of epithelial adherens junctions that are maintained by E-cadherin. H292-shDUOX1 cells also display increased migratory ability, as measured using a recently developed 'donut' cell migration assay ${ }^{32}$ (Figure 1d; Supplementary Figure S1F), and using a scratch wound assay and a Transwell migration assay ${ }^{5}$ (Supplementary Figures S1C and D). In contrast, DUOX1 deletion did not significantly affect proliferation (Figure 1e; Supplementary Figure S1E). Moreover, H292-shDUOX1 cells also demonstrated markedly increased ability of anchorage-independent growth on soft agar (Figure 1f). These findings were consistent across the three separate H292-shDUOX1 clones. Together, these findings suggest that DUOX1 suppression can initiate the process of EMT.
Analysis of various molecular features characteristic of $\mathrm{EMT}^{33}$ revealed that H292-shDUOX1 cells showed markedly reduced expression of E-cadherin, and upregulation of the mesenchymal markers vimentin, collagen and smooth muscle actin, compared with corresponding controls (Figure 1g; Supplementary Figure S1B). Moreover, H292-shDUOX1 cells also displayed constitutive activation of Akt, an important common hallmark of EMT $^{34,35}$ (Supplementary Figure S1B), as well as markedly reduced levels of miR-200b, miR-200c, and miR-205, and enhanced mRNA expression of EMT-promoting transcription factors ZEB1 and ZEB2 (Figure $1 \mathrm{~h}$ ), additional common molecular features of EMT. ${ }^{36,37}$ To confirm the association of DUOX1 silencing with EMT in multiple epithelial cell lines, we performed similar analyses after repetitive siRNA-mediated DUOX1 silencing over a period of 9 days in both H292 cells and the non-cancer bronchial epithelial cell line HBE1, which revealed a similar gradual loss of E-cadherin and induction of mesenchymal markers in both cell models (Supplementary Figures S2A and B). As DUOX1 silencing also resulted in reduced expression of the DUOX1 maturation factor DUOXA1, we determined whether silencing of DUOXA1 itself was able to cause features of EMT. However, similar siRNA targeted suppression of DUOXA1 did not induce loss of E-cadherin, although it did 
a

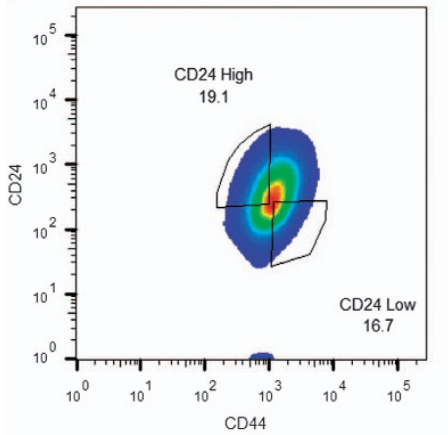

b

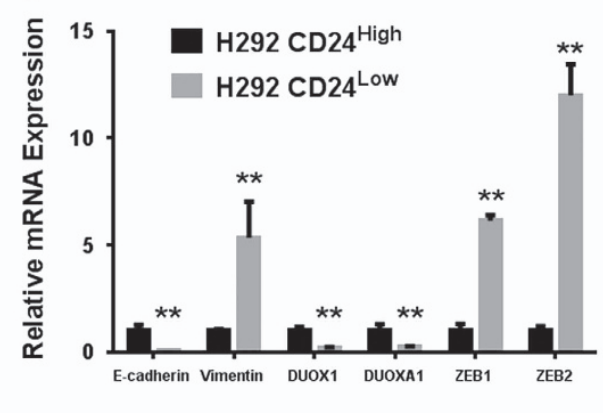

C

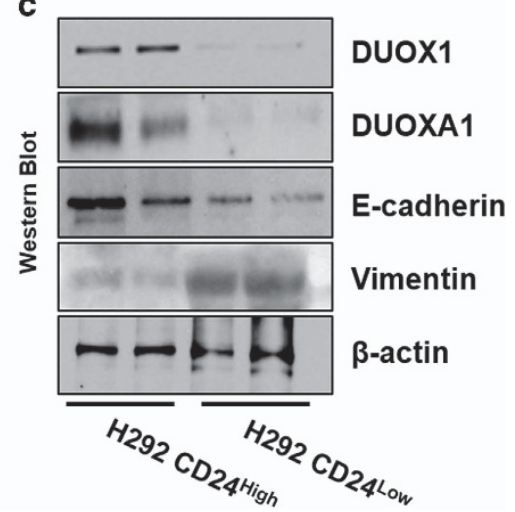

Figure 3. EMT features and loss of DUOX1 in the $\mathrm{H} 292 \mathrm{CD}_{2} 4^{\text {low }}$ sub-population. (a) FACS gating strategy displaying $\mathrm{H} 292$ cell sub-populations were sorted based on their expression of surface antigen CD24/CD44. (b) Quantitative real-time PCR and western blot (c) results displaying $\mathrm{CD} 24^{\text {low }} \mathrm{H} 292$ sub-populations display features of EMT and marked DUOX1 suppression. ${ }^{*} P<0.05$ and ${ }^{* *} P<0.01$ were calculated by Student's $t$-test.

enhance vimentin expression (Supplementary Figure S2C). Finally, induction of EMT features by DUOX1 silencing could be reversed by treatment with the DNA methyltransferase inhibitor 5-aza-deoxycytidine (aza), suggesting that DUOX1 suppression promotes EMT by an epigenetic mechanism (Supplementary Figure S3). Collectively, these findings indicate that loss of DUOX1 expression in lung epithelial cells directly induces loss of epithelial characteristics and promotes development of EMT.

DUOX1 silencing in $\mathrm{H} 292$ cells is associated with enhanced resistance to EGFR tyrosine kinase inhibition

The process of EMT has been associated with acquired resistance of lung cancers to chemotherapeutic drugs and to TKI of the EGFR. $^{38,39}$ Because of the close relationship between DUOX1 and EGFR-dependent signaling, ${ }^{7,8}$ we explored the association between DUOX1 silencing, EMT and sensitivity to the EGFR TKI erlotinib. First, we cultured $\mathrm{H} 292$ cells for prolonged periods of time in the presence of erlotinib to select sub-populations with acquired erlotinib resistance (H292-E90). Consistent with earlier reports, ${ }^{30}$ thus-cultured erlotinib-resistant cells displayed morphological and molecular features of EMT (Figures 2a and b), which were partly reversible by treatment with aza (Supplementary Figure S2). Importantly, compared with normal H292 cells, H292-E90 cells also displayed marked loss of DUOX1 protein and mRNA expression (Figure 2b), and loss of functional DUOX1 activity (Figure 2c), further supporting a close association between DUOX1 silencing and EMT. Also, while normal H292 cells showed impaired growth in the presence of erlotinib, both H292-E90 and H292-shDUOX1 cells displayed marked resistance to erlotinib up to $10 \mu \mathrm{m}$ (Figure 2d), indicating that DUOX1 suppression may be an important determinant of acquired erlotinib resistance in lung cancers. Importantly, to rule out the possibility that enhanced erlotinib resistance may be related to acquired activating mutations in EGFR in our engineered cell lines, we verified by DNA sequencing that neither H292-E90 and H292-shDUOX1 cells contained the following detectable EGFR point mutations at exon 19 D761Y, V769L and N771T or exon 20 T790M or any insertion mutations on exon 19 (D770-N771) (Supplementary Figure S4).

\section{DUOX1 silencing in $\mathrm{H} 292$ cells leads to enhanced CSC} characteristics

One characteristic feature of EMT is altered expression of cell surface markers such as CD44 and CD24, typically involving increased CD44 expression and reduced CD24 expression. ${ }^{30,40}$ As established cancer cell lines are known to display heterogeneity with respect to CD44/CD24 expression, and contain CD $44^{\text {high }} / \mathrm{CD} 24^{\text {low }}$ sub-populations that are typically more mesenchymal in nature, ${ }^{30,40}$ we used fluorescence-activated cell sorting (FACS) high-speed cell sorting to isolate CD24 low $/ C D 44^{\text {high }}$ sub-populations from H292 cells (Figure 3a; Supplementary Figure S5A) and confirmed that this sub-population demonstrated enhanced EMT characteristics compared with CD24 high $/ C D 44^{\text {low }}$ cells. Moreover, CD24 $4^{\text {low }} / C D 44^{\text {high }}$ H292 sub-populations demonstrated marked suppression of DUOX1 mRNA and protein (Figures $3 \mathrm{~b}$ and $\mathrm{c}$; Supplementary Figure S5B), confirming the close relationship between loss of DUOX1 expression and EMT features. Conversely, analysis of H292-shDUOX1 cells as well as H292-E90 cells for mRNA levels and cell surface expression of CD24 and CD44 revealed that both H292-shDUOX1 and H292-E90 cells displayed reduced mRNA expression and cell surface levels of CD24, and enhanced mRNA and surface expression of CD44, compared with corresponding controls (Figures $4 a$ and b; Supplementary Figure S5C), consistent with a shift toward a more mesenchymal phenotype in both cases.

The process of EMT is strongly associated with increased characteristics of CSCs, and CD24 $4^{\text {low }} / \mathrm{CD} 44^{\text {high }}$ sub-populations are thought to display increased CSC features, ${ }^{41}$ suggesting that DUOX1 silencing in lung cancer may also be associated with increased CSC characteristics. To address this, H292-E90 cells and H292-shDUOX1 cells were analyzed for the expression of two commonly accepted lung CSC markers, CD133 and ALDH1, ${ }^{41-43}$ using flow cytometry (Supplementary Figure S6). Indeed, both H292-shDUOX1 and H292-E90 cells were found to contain significantly increased populations of CD133+ cells compared with corresponding controls $(6.61 \pm 0.72 \%$ vs $3.25 \pm 0.70 \%$ and $7.52 \pm 0.85 \%$ vs $2.41 \pm 0.16 \%$, respectively) (Figure $4 \mathrm{c}$ ). Similarly, H292-shDUOX1 cells also displayed an increased fraction of ALDH1+ cells compared with H292-shCTL cells $(23.1 \pm 5.2 \%$ vs $12.5 \pm 0.6 \%)$, although no significant increase was observed in H292-E90 cells (Figure 4d). Finally, culturing of FACS-sorted CD133+ sub-populations in ultra-low attachment plates in stem cell media revealed enhanced growth of CD133+ cells from H292-shDUOX1 or H292-E90 cells compared with their control counterparts (Supplementary Figure S7). Together, these findings demonstrate that DUOX1 silencing in lung cancer is associated with increased CSC properties.

\section{DUOX1 overexpression reverses EMT features}

Although our results indicate that DUOX1 silencing in lung cancer cells promotes EMT and CSC features, the question remains whether these features can also be reversed by DUOX1 overexpression. To address this, we used the alveolar 

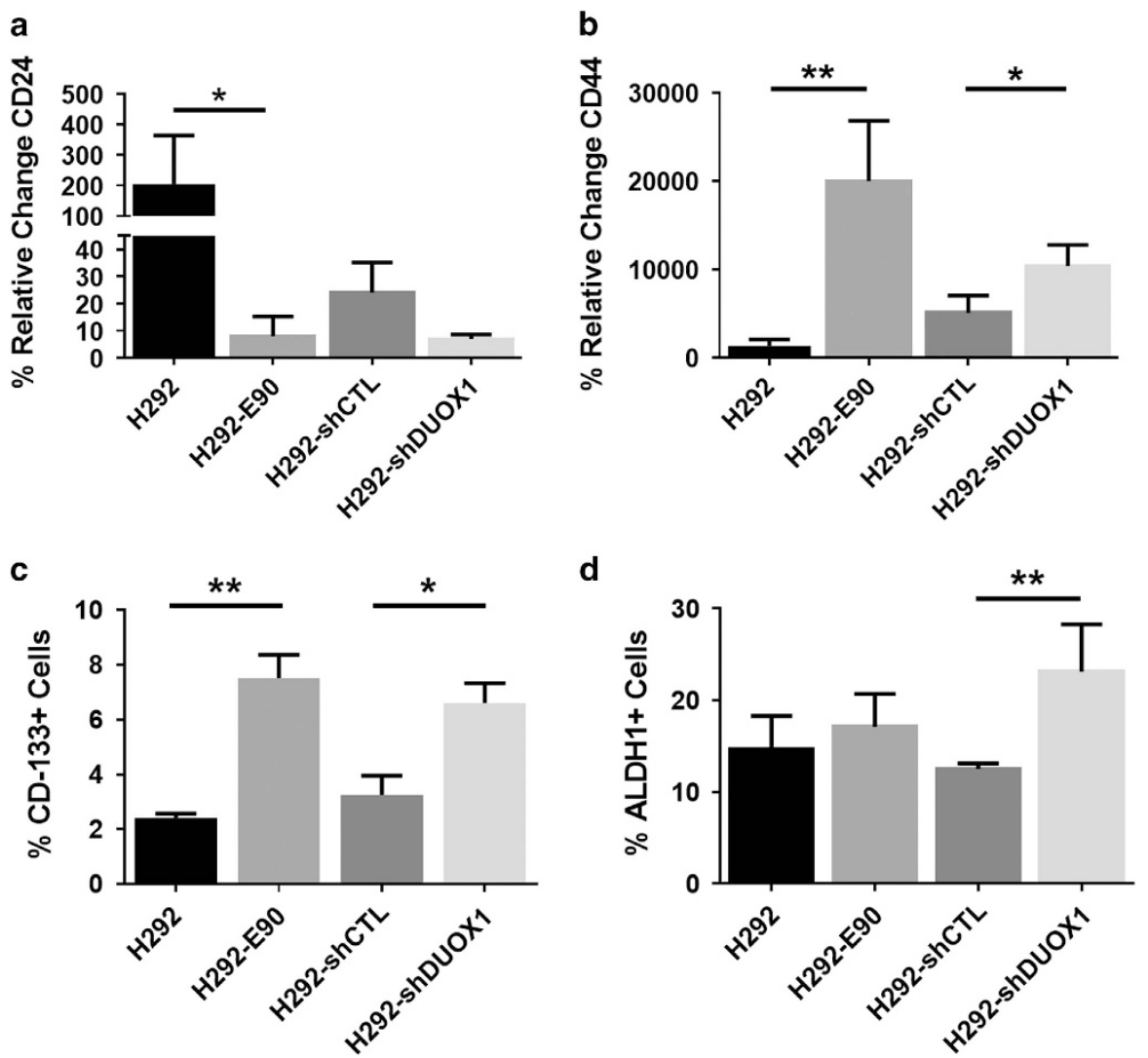

Figure 4. DUOX1 silencing enhances proportions of EMT-like CD24 low $/ C D 44^{\text {high }}$ cell populations and elevates expression of CSC markers CD-133 and ALDH1. (a, b) FACS results of relative percent change of surface antigen CD24 and CD44 (left/right, respectively) vs their unstained controls. (c) FACS results of \%CD-133+ cells as compared with their individual controls. (d) FACS results of \%ALDH1+ cells as compared with their controls. ${ }^{*} P<0.05$ and ${ }^{* *} P<0.01$ were calculated by Student's $t$-test.

adenocarcinoma cell line A549, which displays low DUOX1 and E-cadherin expression and high levels of vimentin, and transfected them with DUOX1 cDNA (A549-pDUOX1; Supplementary Figure S8). As DUOX1 silencing was also associated with suppression of its maturation factor DUOXA1 (Supplementary Figure S2A), we similarly transfected A549 cells with DUOXA1 cDNA (A549-pDUOXA1) for comparison. Remarkably, compared with control A549 cells or A549-pDUOXA1 cells, A549-pDUOX1 cells displayed enhanced epithelial morphology with tight cellcell contacts and increased cobblestone appearance (Figure 5a). Accordingly, DUOX1 overexpression enhanced protein expression of E-cadherin and suppressed expression of the mesenchymal marker vimentin, as visualized by immunofluorescence and western blot analysis (Figures $5 \mathrm{a}$ and b). DUOX1 overexpression in A549 cells also enhanced E-cadherin and reduced vimentin mRNA expression (Supplementary Figure S9). Overexpression of DUOX1, but not DUOXA1, also inhibited migratory capacity of A549 cells in a donut migration assay (Figure 5c; Supplementary Figure S9E) and in a scratch wound assay (Supplementary Figure S8C). Finally, DUOX1 overexpression in A549 cells significantly suppressed their ability to form colonies in soft agar (Figure 5d). Similar reversal of EMT features were observed after overexpression of DUOX1, but not DUOXA1, in the metastatic lung cancer cell line $\mathrm{NCl}-\mathrm{H} 187$ (Supplementary Figure S10) in which DUOX1 is also silenced (Supplementary Figure S1). Collectively, these findings indicate that DUOX 1 overexpression in lung cancer cells can indeed reverse molecular and functional features of EMT.

\section{DUOX silencing promotes tumor invasiveness}

As the process of EMT is strongly linked with increased invasive or metastatic potential of lung cancer cells, ${ }^{33}$ we evaluated whether
DUOX1 silencing was associated with increased invasive/ metastatic behavior. First, H292-shDUOX1 cells and corresponding controls were evaluated in a spheroid invasion assay, which indeed demonstrated significantly increased invasiveness of H292-shDUOX1 cells into the surrounding extracellular matrix, compared with H292-shCTL cells or untransfected control H292 cells (Figure 6a). To address in vivo invasiveness, we injected three clones of H292-shDUOX1 cells, as well as H292-E90 and corresponding control cells, into the tail vein of male CD-1 nude mice and collected major organs after 12 weeks for analysis. Indeed, lung tissues of mice injected with H292-shDUOX1 cells or the EMT cell line H292-E90 displayed altered lung architecture with hypercellularity (Figure $6 \mathrm{~b}$ ) and regions of atypical hyperplasia or small localized neoplasias (Figure 6c; lower panels). No significant abnormalities were observed in livers or spleens (not shown). To evaluate quantitative differences in neoplasias or metastatic lesions within the lung, we sectioned the entire left lung lobe of each animal and examined series of sections separated by about $50 \mu \mathrm{m}$ for the presence of neoplasias with diameter $>120 \mu \mathrm{m}$ (Supplementary Figure S11). Total numbers of counted neoplasias are summarized in Figure $6 \mathrm{~d}$, and indicate that injection of H292-E90 or H292-shDUOX1 (average of all three H292-shDUOX1 clones) resulted in significantly greater numbers of neoplasias compared with their respective controls. Because our histological analysis does not identify the cellular origin of these lung lesions, invasion or engraftment of injected human tumor cells was also evaluated by quantitative PCR analysis of human ALU (hALU) repeat sequences, a DNA element not found in murine tissues. Indeed, mouse lung tissues with altered lung architecture and regions of hyperplasia also contained enhanced levels of hALU DNA (Supplementary Table S1). Although injection 
a
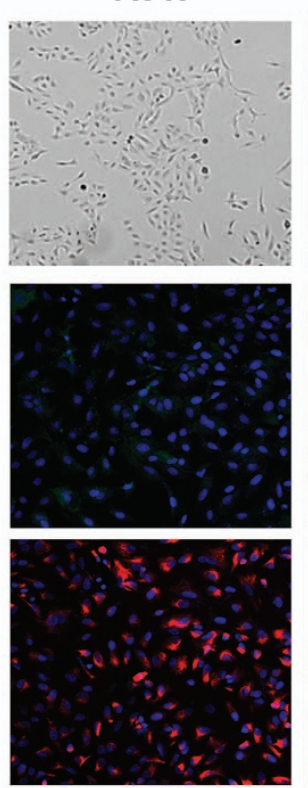

A549-pCTL
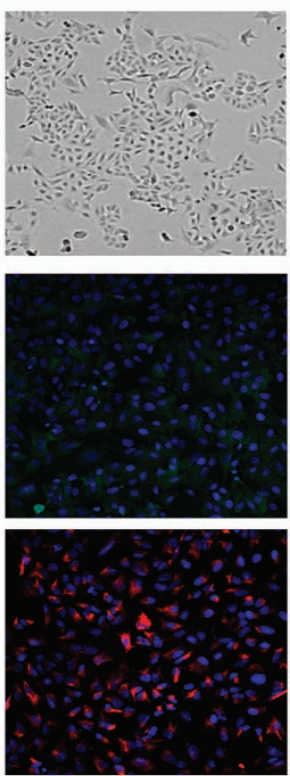

A549-pDUOXA1
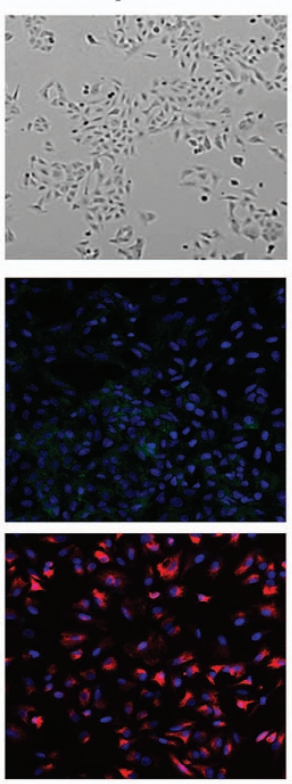

A549-pDUOX1
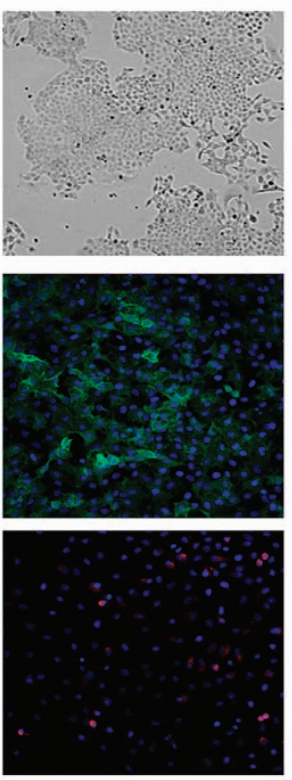

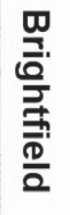

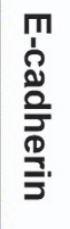

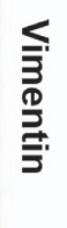

b

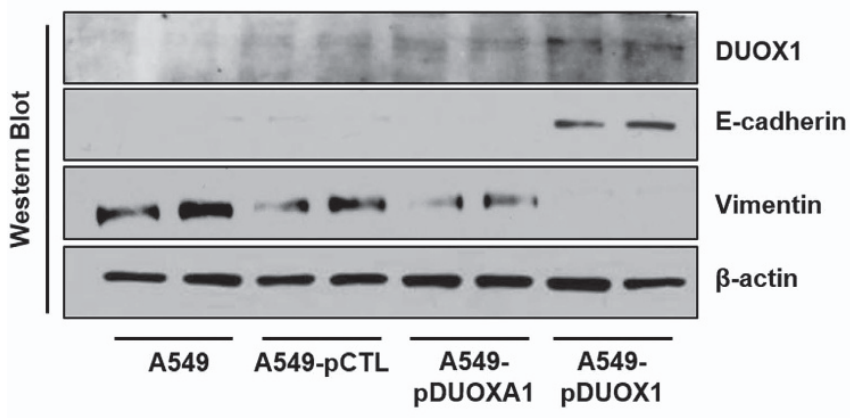

C

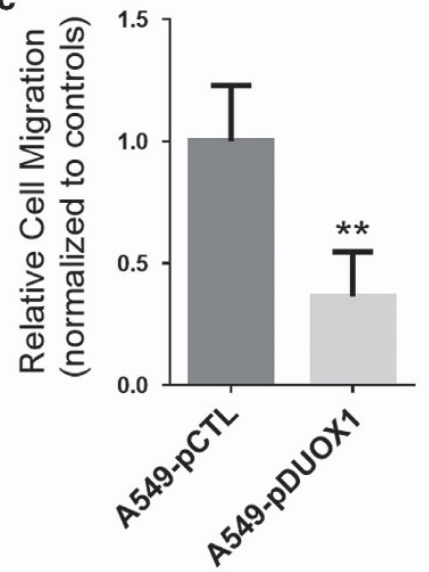

d

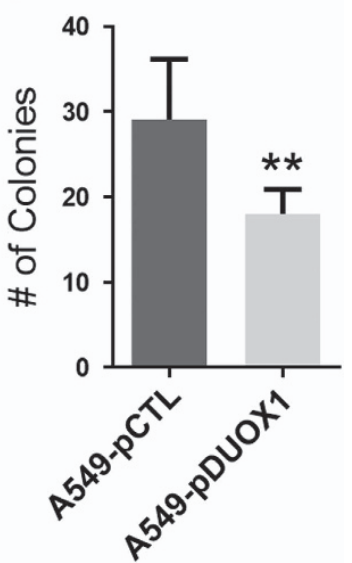

Figure 5. Overexpression of DUOX1 in A549 cells promotes epithelial signatures. (a) Brightfield microscopy reveals DUOX1-overexpressing cells display an epithelial cell morphology. Confocal imaging of epithelial marker E-cadherin and mesenchymal protein vimentin. (b) Western blot analysis shows DUOX1 overexpression promotes features of the mesenchymal-to-epithelial transition. (c) Donut cell migration assay displaying slower migration in DUOX1-overexpressing cells. (d) DUOX1 overexpression inhibits colony formation on soft agar. ${ }^{* * P}<0.01$ was calculated by Student's $t$-test.

of normal H292 or H292-shCTL cells did not induce significant increases in hALU DNA in mouse lungs compared with naive mice $(n=3),>5$-fold increases in hALU DNA were detected in lung tissues after injection of H292-shDUOX1 cells in four out of seven cases, and after injection of H292-E90 (two out of three cases). Overall, these findings indicate that DUOX1 silencing in H292 lung cancer cells significantly enhances invasiveness and metastatic potential.

\section{DISCUSSION}

The main finding of the present studies is that they establish the potential significance of frequent observations of epigenetic silencing of the NADPH oxidase DUOX1 in lung cancer and in other epithelial-derived cancers, ${ }^{18,20,21,44}$ and indicate that DUOX1 silencing is causally linked with the development or progression of EMT, and promotes invasive properties, as well as CSC features and resistance to EGFR TKIs. Our findings may be highly relevant for lung cancer, as the process of EMT is a critical determinant of tumor invasiveness and metastatic potential, and has been frequently linked with poor prognosis. ${ }^{29,33}$ Furthermore, associations of DUOX1 silencing with increased CSC properties and resistance to EGFR inhibitors have important implications for anticancer therapies and suggest that development of approaches aimed at preventing or reversing DUOX1 silencing may be clinically useful in targeting invasive or metastatic lung cancer. Moreover, although EGFR inhibitors are typically not recommended for use in treatment of lung cancers expressing wild-type EGFR, some patients with wild-type EGFR may also benefit, and identification of biomarkers to identify such patients would be desirable. ${ }^{45}$ In this regard, our studies suggest that lung cancers with wild-type EGFR and positive DUOX1 expression may benefit for EGFR inhibitor therapy. The clinical relevance of DUOX1 suppression in lung cancer is also supported by a recent study of patients with hepatocellular carcinoma, which indicated that higher DUOX1 expression strongly correlated with disease-free survival. ${ }^{44}$ However, as our various observations were based on experimental cell models, the clinical relevance of our findings 
a
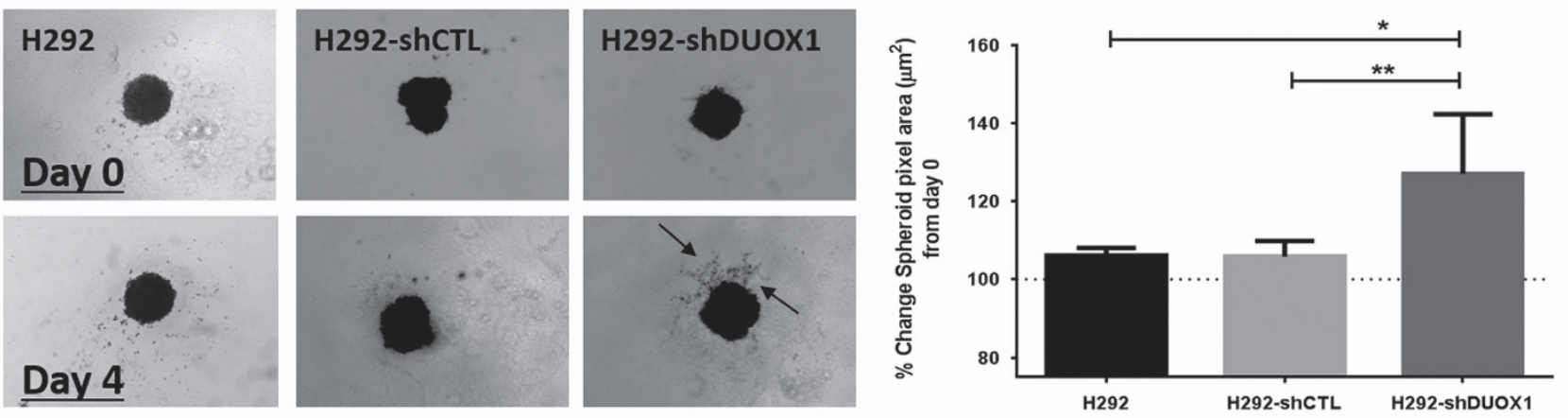

b
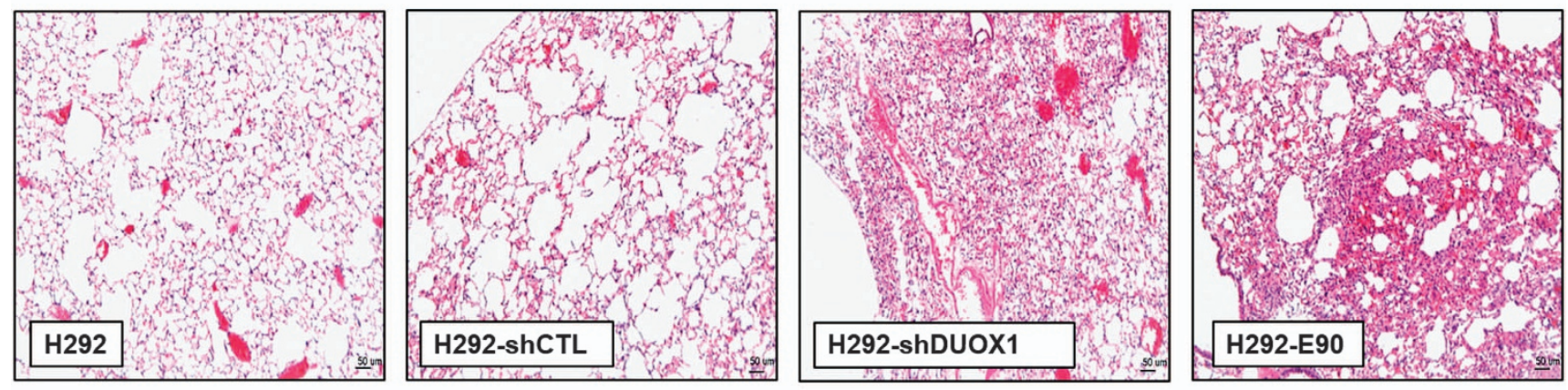

\section{c}
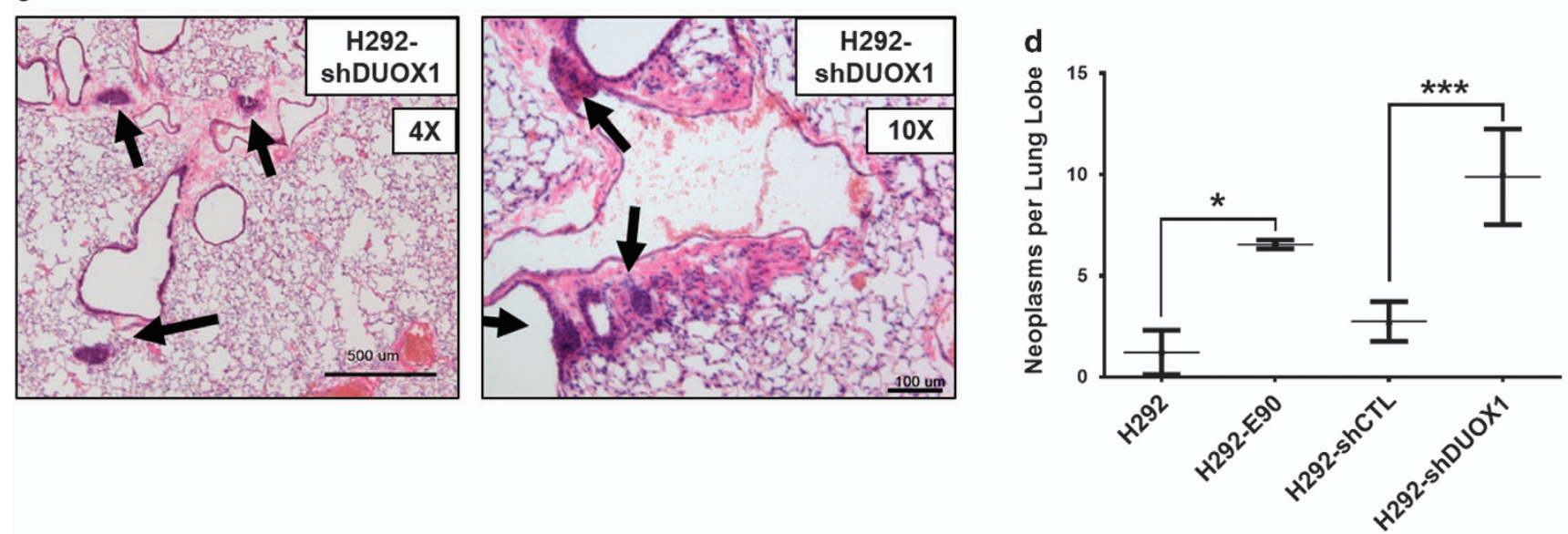

Figure 6. DUOX1 silencing displays enhanced invasive properties. (a) H292-shDUOX1 cell spheroids display invasion into surrounding extracellular matrix (ECM)-like matrix after 4 days, arrows display cellular invasion into mock ECM gel matrix. Right panel shows average percent change in pixel area quantified over the course of multiple experiments. ${ }^{*} P<0.05$, ${ }^{* *} P<0.01$. (b) H\&E-stained lung sections of H292-shDUOX1- or H292-E90-injected mice present significantly altered lung architecture. (c) Localized areas of neoplasias or small engrafted tumors in H292-shDUOX1-injected mouse lungs. (d) Quantification of counted lung neoplasias $>120 \mu \mathrm{m}$ in diameter for animals injected with various cell models. ${ }^{*} P<0.05$ and ${ }^{* * *} P<0.001$ were calculated by Student's $t$-test.

with respect to, for example, tumor stage or progression is still unclear.

Our present findings appear to contrast previous findings by us and others, ${ }^{6,7}$ which indicated that inhibition of DUOX1 in epithelial cells attenuates cell migration in the context of epithelial wound injury responses, which involve the activation of DUOX1 by wound-derived signals such as ATP. The present findings, however, highlight a different scenario related to more gradual changes in epithelial biology and gene expression due to persistent loss of DUOX1, which leads to cell transformation with enhanced intrinsic cell motility, even though cells are less responsive to ATP-mediated wound responses. ${ }^{7}$

In addition to silencing of DUOX1, lung cancers were also found to display silencing of DUOXA $1,{ }^{18}$ a maturation factor for DUOX1, which aids in its trafficking to the plasma membrane to function as a competent NADPH oxidase. ${ }^{2}$ Indeed, our findings indicate that RNAi-dependent silencing of DUOX1 also leads to suppression of DUOXA1 (Supplementary Figure S3A), which might contribute to observed EMT phenomena (for example, Figure 3). Intriguingly, DUOXA1 was originally described as Numb interacting protein (NIP). Numb is a negative regulator of the Notch signaling pathway, which has an important role in airway basal cell differentiation. ${ }^{46}$ Uncontrolled Notch signaling has been associated with metastatic and aggressive epithelial-derived carcinomas, and nearly half of non-small-cell lung cancers display elevated Notch expression/signaling, often associated with suppressed Numb expression. ${ }^{47}$ DUOXA1/NIP expression often mimics the expression status of Numb and loss of Numb has been 
associated with disruption of the epithelial phenotype. ${ }^{48}$ In addition to regulating Numb, NIP/DUOXA1 also has an important role in, for example, myogenesis, with overexpression of NIP/DUOXA1 inhibiting differentiation and promoting apoptosis through enhanced DUOX1-dependent ROS production. ${ }^{49}$ To address the potential involvement of DUOXA1, we used either direct siRNA silencing or overexpression of DUOXA1, which did not significantly affect EMT (Supplementary Figure S2C; Figure 5). Therefore, although some molecular alterations associated with DUOX1 silencing may have resulted from indirect changes in, for example, Notch/Numb status due to suppression of DUOXA1, the observed effects on EMT appear to be primarily due to loss of DUOX1 itself.

The mechanism(s) by which DUOX1 silencing results in increased EMT still remain to be determined. It is plausible that a loss of DUOX1 activation and $\mathrm{H}_{2} \mathrm{O}_{2}$-dependent redox signaling might impact on regulation of Src activation and EGFR-dependent signaling pathways, based on recent studies linking DUOX 1 and Src/EGFR activation in epithelial wound responses. ${ }^{4,70}$ Indeed, expression and activation of Src and EGFR are often increased in lung cancer and form a common therapeutic target in many lung cancers, ${ }^{9,11}$ and acquired resistance to EGFR TKIs may be associated with increased nuclear localization of EGFR. ${ }^{51,52}$ However, our studies did not indicate significant differences in overall ROS production in either DUOX1-deficient $\mathrm{H} 292$ cells $^{7}$ or DUOX1-overexpressing A549 cells under basal conditions (Supplementary Figure S8C), unless DUOX1 was activated by external stimuli such as extracellular ATP. Although we cannot rule out a potential impact of basal DUOX1-derived $\mathrm{H}_{2} \mathrm{O}_{2}$ production in determining cellular outcomes, it is also possible that DUOX1 expression may control cell fate by redox-independent mechanisms, for example, by acting as a scaffold for EGFR or Src, similar to the recently described role of AKAP12. ${ }^{53}$ In addition, it is also plausible that altered DUOX1 status affects cellular redox-dependent mechanisms by more indirect effects, for example, by altered expression of other NOX isozymes. However, although DUOX1 loss or overexpression resulted in some modest alterations in mRNA expression of NOX1, 2 or 4 (Supplementary Figure S12), we did not observe consistent alterations that would point to a major role of other NOX isoforms in our observed outcomes. Future mechanistic studies will be required to address these various issues.

In summary, our findings indicate that DUOX1 silencing, a common finding in epithelial cancers, including lung cancer, ${ }^{12,20,21}$ may be a strong determinant of increased invasive or metastatic potential, as well as resistance to conventional anticancer therapies. At this stage, it is unknown how DUOX1 silencing relates to different types of lung cancer or with specific mutations in EGFR, KRAS and so on. Also, our findings appear to contrast with recent findings indicating a role for DUOX1-derived $\mathrm{H}_{2} \mathrm{O}_{2}$ as a cause of oxidative DNA damage and genomic instability in response to ionizing radiation of the thyroid, as a potential mechanism of development of thyroid cancer. ${ }^{13}$ However, it is plausible initiation of lung carcinogenesis by oxidative events related to DUOX1 may be followed by subsequent epigenetic DUOX1 silencing in established tumors, which in turn may serve to promote invasiveness or metastatic potential. Also, the observed association between DUOX1 expression and sensitivity to EGFR TKI may suggest that positive DUOX1 expression status in lung cancers may be suitable for therapeutic management with EGFR TKI, even if they express wild-type EGFR. Future studies are warranted to further decipher the mechanism(s) involved in epithelial DUOX1 expression and its relation to epithelial phenotype or development of EMT, which would allow for alternative approaches to therapeutically control DUOX1 expression as a potential treatment strategy in established lung cancer.

\section{MATERIALS AND METHODS}

Cell models and transfections

NCl-H292 cells, a human pulmonary mucoepidermoid carcinoma cell line (American Type Culture Collection; ATCC), were propagated in RPMI 1640 medium with $10 \%$ fetal bovine serum $/ 5 \%$ penicillin-streptomycin. DUOX1-deficient H292 cells (H292-shDUOX1) and corresponding vector control cells (H292-shCTL) were generated and maintained as previously described. ${ }^{7}$ Three separate H292-shDUOX1 clones were expanded and utilized in these studies. Erlotinib-resistant sub-clones of H292 cells were generated essentially as described by Yao et al., ${ }^{30}$ by culture for 30 days in the presence of $5 \mu \mathrm{m}$ erlotinib followed by 60 days in the presence of $10 \mu \mathrm{m}$ erlotinib (LC Laboratories, Woburn, MA, USA). Selected resistant $\mathrm{H} 292$ cells (H292-E90) were maintained in the presence of $10 \mu \mathrm{m}$ erlotinib and switched to regular growth media for assays. Alveolar adenocarcinoma cell line A549 cells (ATCC) were cultured according to ATCC guidelines, and grown to $\sim 70 \%$ confluence in a 24-well dish (Costar, Corning, Corning, NY, USA) for transfection with $1 \mu \mathrm{g}$ of CDNA clone of the following vectors: pCMV6-AC-GFP control vector (A549-pCTL) (PS100010, Origene, Rockville, MD, USA); pCMV6-DUOXA1-GFP (A549-pDUOXA1) (RG206754, Origene); or pCMV6-DUOX1-GFP (A549-pDUOX1) (RG223832, Origene). Cells were transfected $2 \times$ over the span of 6 days and then cultured in the presence of $250 \mu \mathrm{g} / \mathrm{ml}$ neomycin (G418 sulfate) (Calbiochem, Millipore, Billerica, MA, USA) for 14 days. Positive transfection of DUOXA1 and/or DUOX1 in A549-pCTL/A549-pDUOXA1/A549-pDUOX1 cell lines was verified by western blot and qRT-PCR analysis.

Other cell lines used in this study include the following: NHBE (normal human bronchial epithelial cells) maintained as described previously; ${ }^{3}$ HBE1 (immortalized human bronchial epithelial cells) maintained as described previously; ${ }^{3,7}$ BEAS2B (human bronchial epithelial cells); Calu-3 (human lung adenocarcinoma cells); $\mathrm{NCl}-\mathrm{H} 1975$ (human lung adenocarcinoma cells); $\mathrm{NCl}-\mathrm{H} 1437$ (human lung adenocarcinoma cells); $\mathrm{NCl}-\mathrm{H} 2170$ (human lung squamous cell carcinoma cells); NCl-H82 (human lung carcinoma cells); $\mathrm{NCl}-\mathrm{H} 460$ (human lung carcinoma cells); and $\mathrm{NCl}-\mathrm{H} 187$ (human lung retinoblastoma cells). Unless indicated otherwise, all cell lines were obtained from ATCC and maintained according to established ATCC protocols, as well as surveyed for mycoplasma contamination.

\section{Trans-epithelial electrical resistance}

Cells were seeded in transwell culture inserts and grown to confluency. Trans-epithelial electrical resistance was measured using an EVOM epithelial voltohmmeter (World Precision Instruments, Sarasota, FL, USA). Trans-epithelial electrical resistance values were corrected for background resistance provided by the insert and/or the media. Data are represented as mean \pm s.d. ${ }^{* *} P<0.01$ were calculated by two-tailed Student's $t$-test. $(n=6$; replicated $2 \times)$.

\section{Anchorage-independent growth}

Six-well culture plates were coated with $2 \mathrm{ml}$ of a $50 / 50$ mixture of $1 \%$ agarose and $2 \times$ growth medium, and appropriate cells were collected, resuspended in 50/50 mixtures of $0.67 \%$ agarose and $2 \times$ complete medium and seeded onto agarose-coated culture plates and placed in a $37^{\circ} \mathrm{C}$ incubator. After 4 weeks, plates were stained with a $0.005 \%$ crystal violet and imaged, and colonies were counted. Data are represented as mean \pm s.d. ${ }^{* *} P<0.01$ were calculated by two-tailed Student's $t$-test. $(n=6$; replicated $2 \times$ ).

In vitro assays of cell migration and invasion

Scratch wound assay. A linear scratch $(\sim 2 \mathrm{~mm})$ was generated in confluent cell monolayers in 24-well culture plates, and detached cells were removed with phosphate-buffered saline (PBS) and wound closure was monitored over a $24 \mathrm{~h}$ period. Wound areas were imaged and analyzed using $\mathrm{NIH}$ Image J software (National Institutes of Health, Bethesda, MD, USA) to determine \% wound closure as an indicator of cell migration. Data are represented as mean \pm s.d. ${ }^{* *} P<0.01$ were calculated by two-tailed Student's $t$-test. $(n=6$; replicated $2 \times)$.

Transwell cell migration assay. Cells were seeded in Boyden-like chambers containing $10 \mathrm{~mm}$ polycarbonate membrane inserts ( $8 \mu \mathrm{m}$ pore size) (Nunc International, Penfield, NY, USA) that were pre-coated with $10 \mu \mathrm{g} / \mathrm{ml}$ fibronectin (Invitrogen). Following incubation for $24 \mathrm{~h}$ at $37^{\circ} \mathrm{C}$, non-migratory cells were removed from membrane with a cotton swab, 
and migrated cells were stained with crystal violet for $30 \mathrm{~min}$ and extracted in $100 \mu \mathrm{l}$ of $0.2 \mathrm{~m}$ sodium acetate buffer $(\mathrm{pH} 4.5)$ for analysis of absorbance at $570 \mathrm{~nm}$. Data are represented as mean \pm s.d. ${ }^{*} P<0.05$ were calculated by two-tailed Student's $t$-test. $(n=6$; replicated $2 \times)$.

Donut cell migration assay. Cells were seeded inside silicone circular gaskets at 10000 cells per donut. Cells were maintained for $\sim 24 \mathrm{~h}$ in complete cell medium to reach full confluency. Donuts were then removed and images were taken immediately following donut removal and $24 \mathrm{~h}$ later. Data were quantified using an ImageJ macro as described and provided previously. ${ }^{32}$ Data are represented as mean \pm s.d. ${ }^{*} P<0.05$ were calculated by two-tailed Student's $t$-test. $(n=12$; replicated $2 \times)$.

In vitro spheroid invasion assay. Invasive properties of the various cell lines were evaluated using a spheroid invasion assay (Cultrex Spheroid Invasion kit; Trevigen, Gaithersburg, MD, USA) according to the manufacturer's instructions. Images were taken on an inverted Olympus IX70 brightfield microscope and subsequently the images were analyzed using $\mathrm{NIH}$ Image J software as defined in Cultrex protocol. Data are represented as mean \pm s.d. ${ }^{*} P<0.05 ;{ }^{* *} P<0.01$ were calculated by one-way analysis of variance $(n=18$; replicated $2 \times)$.

\section{Western blot analysis}

Cells cultured in 24-well culture plates were lysed using $100 \mu \mathrm{l}$ of $1 \times$ western solubilization lysis buffer (1\% Triton, $50 \mathrm{~mm}$ HEPES, $250 \mathrm{~mm}$ $\mathrm{NaCl}, 10 \%$ glycerol, $1.5 \mathrm{~mm} \mathrm{MgCl}, 1 \mathrm{~mm}$ phenylmethylsulfonyl fluoride, $1 \mathrm{~mm}$ EGTA, $2 \mathrm{~mm} \mathrm{Na} \mathrm{VO}_{4}, 10 \mu \mathrm{g} / \mathrm{ml}$ aprotinin and $10 \mu \mathrm{g} / \mathrm{ml}$ leupeptin; $\mathrm{pH}$ 7.4) per well. Equal amounts of protein (20-25 $\mu \mathrm{g}$; determined using bicinchoninic acid protein assay) were separated on Novex $10 \%$ Tris-Glycine gels (Life Technologies, Grand Island, NY, USA), transferred to nitrocellulose membranes and blotted using antibodies against the following: vimentin (\#5741; 1:500; Cell Signaling, Danvers, MA, USA); Akt (\#9272; 1:1000; Cell Signaling); p-Akt (\#4060X; 1:1000; Cell Signaling); $\beta$-actin (\#MA5-15739; 1:10 000; Invitrogen, Waltham, MA, USA); Collagen (\#ab34710; 1:500; Abcam, Cambridge, MA, USA); E-cadherin (\#3195; 1:1000; Cell Signaling); DUOX1 (1:1000; kindly provided by F. Miot $\left.{ }^{3,54}\right)$; and DUOXA1 (\#H00090527-B01P; 1:1000; Abnova, Jhongli, Taiwan), and detected using enhanced chemiluminescence (Pierce, Rockfort, IL, USA).

\section{Immunohistochemical analysis and morphological imaging}

H292, H292-shCTL, H292-shDUOX1 and H292-E90 cells were seeded in eight-well glass chamber slides, fixed with $4 \%$ paraformaldehyde and permeabilized with $0.2 \%$ Triton $\mathrm{X}-100$ in $1 \%$ bovine serum albumin (BSA) in PBS for $15 \mathrm{~min}$. Slides were washed $2 \times$ with $1 \%$ BSA/PBS solution, blocked with $10 \%$ goat serum for $1 \mathrm{~h}$ and then subjected to staining with antibodies against E-cadherin (1:100; Invitrogen), vimentin (1:100; Cell Signaling) or a-smooth muscle actin (1:100; Cell Signaling). Nuclei were counterstained with 4,6-diamidino-2-phenylindole $(10 \mu \mathrm{g} / \mathrm{ml}$ in $1 \%$ BSA/PBS). Cells were washed $2 \times$ with $1 \%$ BSA/PBS and mounted with glass coverslip. Images were taken on a Zeiss LSM 510 META laser scanning confocal microscope (Zeiss, Jena, Germany). Brightfield images were taken using a Zeiss Interskop phase contrast microscope interfaced with a digital camera (Zeiss).

\section{RNA interference}

Cells were grown to $\sim 70 \%$ confluence in 24-well plates and transfected with $0.1 \mu \mathrm{m}$ siRNA targeted against DUOX1 mRNA (\#117546, Ambion siRNA, Life Technologies; accession No. NM_017434.3/NM_175940.1), DUOXA1 mRNA (\#90527, Dharmacon, GE, Lafayette, CO, USA; accession No. NM_144565.2) or $0.1 \mu \mathrm{M}$ non-targeting control siRNA (Dharmacon, GE) in RPMI 1640 media (Gibco, Life Technologies), and incubated for $24 \mathrm{~h}$ at $37^{\circ} \mathrm{C}$. Media then replaced with complete media, and cells were incubated for an additional $48 \mathrm{~h}$ and then subjected to one or two successive similar transfections, and then collected (3, 6 or 9 days after initial transfection for extraction of miRNA, RNA or protein).

Analysis of cell viability and proliferation

Cells were seeded at $1 \times 10^{4}$ per well into a 96-well black flat bottom fluorescence plates (Nunc International, Rochester, NY, USA) and cultured in RPMI 1640 (no fetal bovine serum) in the presence of various doses of erlotinib (0.1-10 $\mu \mathrm{m}$; LC Laboratories), by exchanging media \pm erlotinib daily for up to 3 days, after which cell viability was determined using ATP
TiterGlo assay reagent (Promega, Madison, WI, USA) Data are represented as mean \pm s.d. ${ }^{*} P<0.05 ;{ }^{* *} P<0.01$ were calculated by one-way analysis of variance $(n=6$; replicated $2 \times)$. Cell proliferation was analyzed by cell seeding at various densities and culture in full growth media for up to $72 \mathrm{~h}$, using ATP TiterGlo reagent. Data are represented as mean \pm s.d. Data did not reach significance $(P<0.05)$ as tested by a two-tailed Student's $t$-test $(n=6$; replicated $2 \times)$. Additional cell proliferation assays were performed by seeding cells at equal cell densities and collected daily for 7 days, and counted to assess proliferation. Data are represented as mean \pm s.d. Data did not reach significance $(P<0.05)$ as tested by a two-tailed Student's $t$-test $(n=6$; replicated $2 \times)$.

\section{RNA extraction, $P C R$ and quantitative real-time $P C R$}

Total RNA was extracted with GeneJET RNA purification kit (Thermo Scientific, Waltham, MA, USA). For mature miRNA extraction, miRNeasy mini kit was utilized (Qiagen, Valencia, CA, USA). RNA extracts were reverse-transcribed and real-time PCR was performed using SYBR green quantitative PCR assays. All quantitative PCR primers were purchased from Sigma-Aldrich (St Louis, MO, USA) unless otherwise noted. Mature miRNA was reverse-transcribed utilizing miScript II RT Kit, and subsequent real-time PCR was performed with miScript SYBR Green PCR Kit and miScript Primer Assays (Qiagen). Data are represented as mean \pm s.d. ${ }^{*} P<0.05 ;{ }^{* *} P<0.01$ were calculated by two-tailed Student's $t$-test or oneway analysis of variance $(n=6$; replicated $2 \times)$.

\section{Evaluation of CSC characteristics}

CSC markers were evaluated by multi-color flow cytometric analysis (FACS) on a BD FACS LSRII (BD Biosciences, San Jose, CA, USA), after cell staining with $0.5 \mu \mathrm{g}$ per $100 \mu \mathrm{l}$ of anti-CD24-Pacific Blue (eBioscience, San Diego, CA, USA) and anti-CD44-Alexafluor 700 (Biolegend, San Diego, CA, USA), $0.5 \mu \mathrm{g}$ per $100 \mu \mathrm{l}$ of anti-human-CD133-AF700 (MACS, Auburn, CA, USA) or ALDEFLUOR reagent $(0.5 \mu \mathrm{g}$ per $100 \mu \mathrm{l}$; Stem Cell Technologies, Vancouver, BC, CA). Data are represented as mean \pm s.d. ${ }^{*} P<0.05$; ${ }^{* *} P<0.01$ were calculated by two-tailed Student's t-test $(n=6 ;$ replicated $2 \times)$. CD24 ${ }^{\text {low }} / \mathrm{CD}_{24} 4^{\text {high }}$ sub-populations of $\mathrm{NCl}-\mathrm{H} 292$ cells were sorted utilizing anti-CD24-Pacific Blue antibody, using a BD FACSAria high-speed cell sorter (BD Biosciences). Similarly, CD133+ cells were sorted and collected in high fetal bovine serum (20\%)-containing RPMI-1640 cell media (Gibco, Grand Island, NY) supplemented with $5 \%$ penicillin/streptomycin. Cells were centrifuged, washed $2 \times$ with $1 \times$ PBS, and resuspended in StemPro mesenchymal stem cell growth media supplemented with StemPro MSC SFM supplement (Gibco) and placed in ultra-low attachment plates (Corning) and monitored over the course of 7 days.

\section{In vivo tumor metastasis}

Charles River CD1 nude male mice (8 weeks) were injected into the tail vein with $1 \times 10^{6}$ of various cell lines (H292, H292-shCTL, H292-shDUOX1 (three separate clones) or H292-E90) in $1 \times$ sterile PBS, at random. Mice were weighed twice weekly and monitored for overall health up until 12 weeks post injection, after which mice were killed and tissues collected. Lung sections were cut at a thickness of $5 \mu \mathrm{m}$ and stained with hematoxylin and eosin (H\&E) for visual observation of lung structure. To quantify total numbers of neoplasias, lung tissue blocks were cut at a thickness of $5 \mu \mathrm{m}$ and stained for H\&E, then tissue blocks were cut into $50 \mu \mathrm{m}$, and another $5 \mu \mathrm{m}$ section was isolated for H\&E staining. This process was repeated until the tissue block was exhausted. Each tissue section was examined and neoplasias $>120 \mu \mathrm{m}$ were counted. Neoplasia size was determined by measurements taken on Olympus BX-50 imaging software (Olympus, Center Valley, PA, USA). Neoplasm quantification was not performed under blinded conditions. Data are represented as mean \pm s.d. ${ }^{*} P<0.05 ;{ }^{* * *} P<0.001$ were calculated by two-tailed Student's $t$-test (for H292, H292-E90 and H292-shCTL, $n=3$ each; for H292-shDUOX1, $n=7$ ). Genomic DNA from mouse lungs was isolated utilizing phenolchloroform extraction method and $30 \mathrm{ng}$ of DNA was further amplified by quantitative PCR for human ALU DNA sequences. ${ }^{55}$ All animal experiments were performed in compliance with IACUC regulations and guidelines.

\section{Analysis of DUOX1 activity}

DUOX1 activity was evaluated by extracellular $\mathrm{H}_{2} \mathrm{O}_{2}$ production in response to cell stimulation with $100 \mu \mathrm{M}$ ATP (Sigma-Aldrich), as described previously. Data are represented as mean \pm s.d. ${ }^{* *} P<0.01$ were calculated by two-tailed Student's $t$-test $(n=6$; replicated $2 \times)$. 


\section{Analysis of EGFR mutations}

To determine potential acquisition of EGFR mutations in various engineered cell lines, CDNA was PCR amplified for EGFR exon 18 (5'-GCT GAGGTGACCCTTGTCTC-3' (F), 5'-ACAGCTTGCAAGGACTCTGG-3' (R)), EGFR exon 19 (5'-GCTGGTAACATCCACCCAGA-3' (F), 5'-GAGAAAAGGTGGGCCT GAG-3' (R)), EGFR exon 20 (5'-CCTCCTTCTGGCCACCATGCG-3' (F), 5'-CATGT GAGGATCCTGGCTCC-3' (R)) and EGFR exon 21 (5'-CGGATGCAGAGCTTC TTCCC-3' (F), 5'-AGGCAGCCTGGTCCCTGGTG-3' (R)), purified and prepared for Sanger sequencing utilizing the forward (F) primers listed above. Samples were analyzed on an ABI Prism 3130xl Genetic Analyzer (Thermo Scientific) at the University of Vermont DNA Analysis Core Facility.

\section{Statistical analysis}

All experimental procedures are reported with $\geqslant 4$ individual replicates and replicated experimentally at a minimum of two times. All data are subjected to either students two-tailed $t$-test or a one way analysis of variance for the determination of statistical significance based on the set being analyzed. $P$-values $<0.05\left({ }^{*}\right), P$-values $<0.01(* *)$. Correction for multiple comparisons were made using either Welch's correction or the Holm-Sidak method depending on the data set. All data were calculated in Microsoft Excel 2010, Graphpad prism 6 or NIH ImageJ.

\section{CONFLICT OF INTEREST}

The authors declare no conflict of interest.

\section{ACKNOWLEDGEMENTS}

We thank Dr Umadevi Wesley (University of Wisconsin) for providing DUOX1 expression vectors, and Dr Jeffrey Spees (UVM) for the hALU probe and primer. We thank Thomm Buttolph and Dr Sheryl White of the UVM Cellular and Molecular Biology Core for amplification and purification of DUOX1 overexpression vectors; Tim Hunter and Jessica Hoffman of the UVM Cancer Center DNA Analysis Facility; Dr Roxana del Rio in the UVM Flow Cytometry Core; and Dr Douglas Taatjes, Nicole Bishop and Nicole Bouffard of the UVM Microscopy Imaging Center. We thank Dr Alan Howe at UVM for providing silicone gaskets for the Donut cell migration assay. This research was supported by NIH RO1 HL085646 (AvdV), F32 HL129706 (DEH), an internal grant from the UVM College of Medicine (AvdV) and a J. Walter Juckett Scholarship Award from the Lake Champlain Cancer Research Organization (LCCRO). ACL is supported by a T32 training fellowship (HL076122) in the Vermont Lung Center.

\section{REFERENCES}

1 Leto TL, Geiszt M. Role of Nox family NADPH oxidases in host defense. Antioxid Redox Signal 2006; 8: 1549-1561.

2 Fischer $\mathrm{H}$. Mechanisms and function of DUOX in epithelia of the lung. Antioxid Redox Signal 2009; 11: 2453-2465.

3 Hristova M, Habibovic A, Veith C, Janssen-Heininger YM, Dixon AE, Geiszt $M$ et al. Airway epithelial dual oxidase 1 mediates allergen-induced IL-33 secretion and activation of type 2 immune responses. J Allergy Clin Immunol 2016; 137: 1545-1556.

4 Gorissen SH, Hristova M, Habibovic A, Sipsey LM, Spiess PC, Janssen-Heininger YM et al. Dual oxidase-1 is required for airway epithelial cell migration and bronchiolar reepithelialization after injury. Am J Respir Cell Mol Biol 2013; 48: 337-345.

5 Wesley UV, Bove PF, Hristova M, McCarthy S, van der Vliet A. Airway epithelial cell migration and wound repair by ATP-mediated activation of dual oxidase 1. $J$ Biol Chem 2007; 282: 3213-3220.

6 Yoo SK, Freisinger CM, LeBert DC, Huttenlocher A. Early redox, Src family kinase, and calcium signaling integrate wound responses and tissue regeneration in zebrafish. J Cell Biol 2012; 199: 225-234.

7 Sham D, Wesley UV, Hristova M, van der Vliet A. ATP-mediated transactivation of the epidermal growth factor receptor in airway epithelial cells involves DUOX1-dependent oxidation of Src and ADAM17. PLOS ONE 2013; 8: e54391.

8 Koff JL, Shao MX, Ueki IF, Nadel JA. Multiple TLRs activate EGFR via a signaling cascade to produce innate immune responses in airway epithelium. Am J Physiol Lung Cell Mol Physiol 2008; 294: L1068-L1075.

9 Reungwetwattana T, Dy GK. Targeted therapies in development for non-small cell lung cancer. J Carcinog 2013; 12: 22.

10 Summy JM, Gallick GE. Src family kinases in tumor progression and metastasis. Cancer Metastasis Rev 2003; 22: 337-358.

11 Kim LC, Song L, Haura EB. Src kinases as therapeutic targets for cancer. Nat Rev Clin Oncol 2009; 6: 587-595.
12 Meitzler JL, Antony S, Wu Y, Juhasz A, Liu H, Jiang G et al. NADPH oxidases: a perspective on reactive oxygen species production in tumor biology. Antioxid Redox Signal 2014; 20: 2873-2889.

13 Ameziane-El-Hassani R, Talbot M, de Souza Dos Santos MC, Al Ghuzlan A, Hartl D, Bidart JM et al. NADPH oxidase DUOX1 promotes long-term persistence of oxidative stress after an exposure to irradiation. Proc Natl Acad Sci USA 2015; 112: 5051-5056.

14 Block K, Gorin Y. Aiding and abetting roles of NOX oxidases in cellular transformation. Nat Rev Cancer 2012; 12: 627-637.

15 Kamata T. Roles of Nox1 and other Nox isoforms in cancer development. Cancer Sci 2009; 100: 1382-1388.

16 Gius D, Spitz DR. Redox signaling in cancer biology. Antioxid Redox Signal 2006; 8: 1249-1252.

17 Trachootham D, Alexandre J, Huang P. Targeting cancer cells by ROS-mediated mechanisms: a radical therapeutic approach? Nat Rev Drug Discov 2009; 8: 579-591.

18 Luxen S, Belinsky SA, Knaus UG. Silencing of DUOX NADPH oxidases by promoter hypermethylation in lung cancer. Cancer Res 2008; 68: 1037-1045.

19 Wu BK, Mei SC, Brenner C. RFTS-deleted DNMT1 enhances tumorigenicity with focal hypermethylation and global hypomethylation. Cell Cycle 2014; 13: 3222-3231.

20 Juhasz A, Ge Y, Markel S, Chiu A, Matsumoto L, van Balgooy J et al. Expression of NADPH oxidase homologues and accessory genes in human cancer cell lines, tumours and adjacent normal tissues. Free Radic Res 2009; 43: 523-532.

21 Ling Q, Shi W, Huang C, Zheng J, Cheng Q, Yu K et al. Epigenetic silencing of dual oxidase 1 by promoter hypermethylation in human hepatocellular carcinoma. Am J Cancer Res 2014; 4: 508-517.

22 Takahashi A, Ohtani N, Yamakoshi K, lida S, Tahara H, Nakayama K et al. Mitogenic signalling and the p16INK4a-Rb pathway cooperate to enforce irreversible cellular senescence. Nat Cell Biol 2006; 8: 1291-1297.

23 Ogasawara MA, Zhang $\mathrm{H}$. Redox regulation and its emerging roles in stem cells and stem-like cancer cells. Antioxid Redox Signal 2009; 11: 1107-1122.

24 Zhou D, Shao L, Spitz DR. Reactive oxygen species in normal and tumor stem cells. Adv Cancer Res 2014; 122: 1-67.

25 Ren S, Su C, Wang Z, Li J, Fan L, Li B et al. Epithelial phenotype as a predictive marker for response to EGFR-TKIs in non-small cell lung cancer patients with wild-type EGFR. Int J Cancer 2014; 135: 2962-2971.

26 Thomson S, Buck E, Petti F, Griffin G, Brown E, Ramnarine N et al. Epithelial to mesenchymal transition is a determinant of sensitivity of non-small-cell lung carcinoma cell lines and xenografts to epidermal growth factor receptor inhibition. Cancer Res 2005; 65: 9455-9462.

27 Yauch RL, Januario T, Eberhard DA, Cavet G, Zhu W, Fu L et al. Epithelial versus mesenchymal phenotype determines in vitro sensitivity and predicts clinical activity of erlotinib in lung cancer patients. Clin Cancer Res 2005; 11: 8686-8698.

28 Xiao D, He J. Epithelial mesenchymal transition and lung cancer. J Thorac Dis 2010; 2: 154-159.

29 Nurwidya F, Takahashi F, Murakami A, Takahashi K. Epithelial mesenchymal transition in drug resistance and metastasis of lung cancer. Cancer Res Treat 2012; 44: 151-156.

30 Yao Z, Fenoglio S, Gao DC, Camiolo M, Stiles B, Lindsted T et al. TGF-beta IL-6 axis mediates selective and adaptive mechanisms of resistance to molecular targeted therapy in lung cancer. Proc Natl Acad Sci USA 2010; 107: 15535-15540.

31 Shen BQ, Finkbeiner WE, Wine JJ, Mrsny RJ, Widdicombe JH. Calu-3: a human airway epithelial cell line that shows CAMP-dependent $\mathrm{Cl}$ - secretion. Am J Physiol 1994; 266: L493-L501.

32 McKenzie AJ, Campbell SL, Howe AK. Protein Kinase A activity and anchoring are required for ovarian cancer cell migration and invasion. PLOS ONE 2011; 6: e26552.

33 Kalluri R, Weinberg RA. The basics of epithelial-mesenchymal transition. J Clin Invest 2009; 119: 1420-1428.

34 Larue L, Bellacosa A. Epithelial-mesenchymal transition in development and cancer: role of phosphatidylinositol 3' kinase/AKT pathways. Oncogene 2005; 24: 7443-7454.

35 Lamouille S, Derynck R. Emergence of the phosphoinositide 3-kinase-Aktmammalian target of rapamycin axis in transforming growth factor-betainduced epithelial-mesenchymal transition. Cells Tissues Organs 2011; 193: 8-22.

36 Brabletz S, Brabletz T. The ZEB/miR-200 feedback loop-a motor of cellular plasticity in development and cancer? EMBO Rep 2010; 11: 670-677.

37 Wellner U, Schubert J, Burk UC, Schmalhofer O, Zhu F, Sonntag A et al. The EMT-activator ZEB1 promotes tumorigenicity by repressing stemness-inhibiting microRNAs. Nat Cell Biol 2009; 11: 1487-1495.

38 Shien K, Toyooka S, Yamamoto H, Soh J, Jida M, Thu KL et al. Acquired resistance to EGFR inhibitors is associated with a manifestation of stem cell-like properties in cancer cells. Cancer Res 2013; 73: 3051-3061. 
39 Thomson S, Petti F, Sujka-Kwok I, Epstein D, Haley JD. Kinase switching in mesenchymal-like non-small cell lung cancer lines contributes to EGFR inhibitor resistance through pathway redundancy. Clin Exp Metastasis 2008; 25: 843-854.

40 Singh A, Settleman J. EMT, cancer stem cells and drug resistance: an emerging axis of evil in the war on cancer. Oncogene 2010; 29: 4741-4751.

41 Eramo A, Lotti F, Sette G, Pilozzi E, Biffoni M, Di Virgilio A et al. Identification and expansion of the tumorigenic lung cancer stem cell population. Cell Death Differ 2008; 15: 504-514.

42 Jiang F, Qiu Q, Khanna A, Todd NW, Deepak J, Xing L et al. Aldehyde dehydrogenase 1 is a tumor stem cell-associated marker in lung cancer. Mol Cancer Res 2009; 7: 330-338.

43 Sarvi S, Mackinnon AC, Avlonitis N, Bradley M, Rintoul RC, Rassl DM et al. CD133+ cancer stem-like cells in small cell lung cancer are highly tumorigenic and chemoresistant but sensitive to a novel neuropeptide antagonist. Cancer Res 2014; 74: 1554-1565.

44 Chen SS, Yu KK, Ling QX, Huang C, Li N, Zheng JM et al. The combination of three molecular markers can be a valuable predictive tool for the prognosis of hepatocellular carcinoma patients. Sci Rep 2016; 6: 24582.

45 Laurie SA, Goss GD. Role of epidermal growth factor receptor inhibitors in epidermal growth factor receptor wild-type non-small-cell lung cancer. J Clin Oncol 2013; 31: 1061-1069.

46 Rock JR, Gao X, Xue Y, Randell SH, Kong YY, Hogan BL. Notch-dependent differentiation of adult airway basal stem cells. Cell Stem Cell 2011; 8: 639-648.

47 Westhoff B, Colaluca IN, D'Ario G, Donzelli M, Tosoni D, Volorio S et al. Alterations of the Notch pathway in lung cancer. Proc Natl Acad Sci USA 2009; 106: 22293-22298.

48 Ostrakhovitch EA. Interplay between Numb and Notch in epithelial cancers: role for dual oxidase maturation factor. Eur J Cancer 2009; 45: 2071-2076.
49 Sandiford SD, Kennedy KA, Xie X, Pickering JG, Li SS. Dual oxidase maturation factor 1 (DUOXA1) overexpression increases reactive oxygen species production and inhibits murine muscle satellite cell differentiation. Cell Commun Signal 2014; 12: 5 .

50 Heppner DE, van der Vliet A. Redox-dependent regulation of epidermal growth factor receptor signaling. Redox Biol. 2016; 8: 24-27.

51 Li C, lida M, Dunn EF, Ghia AJ, Wheeler DL. Nuclear EGFR contributes to acquired resistance to cetuximab. Oncogene 2009; 28: 3801-3813.

52 Brand TM, lida M, Luthar N, Starr MM, Huppert EJ, Wheeler DL. Nuclear EGFR as a molecular target in cancer. Radiother Oncol 2013; 108: 370-377.

53 Su B, Gao L, Meng F, Guo LW, Rothschild J, Gelman IH. Adhesion-mediated cytoskeletal remodeling is controlled by the direct scaffolding of Src from FAK complexes to lipid rafts by SSeCKS/AKAP12. Oncogene 2013; 32: 2016-2026.

54 De Deken X, Wang D, Many MC, Costagliola S, Libert F, Vassart G et al. Cloning of two human thyroid cDNAs encoding new members of the NADPH oxidase family. J Biol Chem 2000; 275: 23227-23233.

55 Munoz JR, Stoutenger BR, Robinson AP, Spees JL, Prockop DJ. Human stem/progenitor cells from bone marrow promote neurogenesis of endogenous neural stem cells in the hippocampus of mice. Proc Natl Acad Sci USA 2005; 102: $18171-18176$.

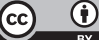

Oncogenesis is an open-access journal published by Nature Publishing Group. This work is licensed under a Creative Commons Attribution 4.0 International License. The images or other third party material in this article are included in the article's Creative Commons license, unless indicated otherwise in the credit line; if the material is not included under the Creative Commons license, users will need to obtain permission from the license holder to reproduce the material. To view a copy of this license, visit http://creativecommons.org/licenses/by/4.0/

(c) The Author(s) 2016

Supplementary Information accompanies this paper on the Oncogenesis website (http://www.nature.com/oncsis). 